\title{
Spectral characterizations of sun graphs and broken sun graphs *
}

\author{
Romain Boulet ${ }^{\dagger}$
}

10 Dec 2009

\begin{abstract}
Several matrices can be associated to a graph such as the adjacency matrix or the Laplacian matrix. The spectrum of these matrices gives some informations about the structure of the graph and the question "Which graphs are determined by their spectrum?" remains a difficult problem in algebraic graph theory. In this article we enlarge the known families of graphs determined by their spectrum by considering some unicyclic graphs. An odd (resp. even) sun is a graph obtained by appending a pendant vertex to each vertex of an odd (resp. even) cycle. A broken sun is a graph obtained by deleting pendant vertices of a sun. In this paper we prove that a sun is determined by its Laplacian spectrum, an odd sun is determined by its adjacency spectrum (counter-examples are given for even suns) and we give some spectral characterizations of broken suns.

keywords: Graphs, algebraic graph theory, spectral graph theory, unicyclic graphs, Laplacian matrix, adjacency matrix, graphs determined by their spectrum, sun graphs.
\end{abstract}

\section{Introduction}

Several matrices can be associated to a graph such as the adjacency matrix (denoted by $A$ ) or the Laplacian matrix $L=D-A$ where $D$ is the diagonal matrix of degrees. Some structural properties can be deduced from their spectrum but in general we can't determine a graph from its adjacency or Laplacian spectrum. A natural question arise "Which graphs are determined by their spectrum?" [6]. Two graphs are $A$-cospectral (resp. $L$-cospectral) if they have the same $A$-spectrum (resp. $L$-spectrum). Some papers study spectral properties of unicyclic graphs (see [5, 1 for instance) but few is done to find families of unicyclic graphs determined by their spectrum (see 10, 3, for examples of unicyclic graphs determined by their spectrum).

The Sun on $n=2 p$ vertices, denoted by $S_{u n}$, is the graph obtained by appending a pendant vertex to each vertex of a $p$-cycle. A broken sun is a

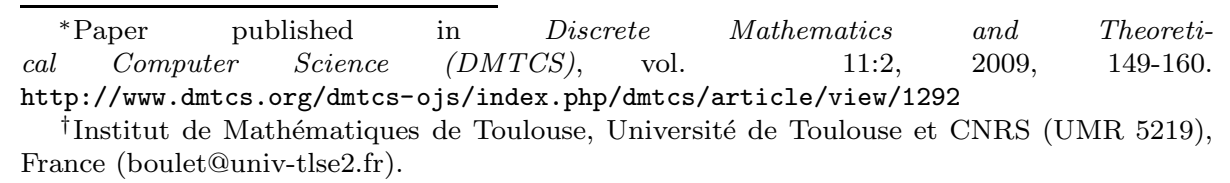


connected unicyclic subgraph of a sun. We denote by $B S(p, q)$ the set of broken suns with $n=p+q$ vertices and with a $p$-cycle; note that $B S(p, p)=\left\{S u n_{p}\right\}$. For $p>2$ and $0<q<p$, a consecutive broken sun, denoted by $C B S$ sun $_{p, q}$, is the graph belonging to $B S(p, q)$ such that the subgraph induced by the vertices of degree 2 is a path on $p-q$ vertices. Some examples are depicted in Figures 1. 2 and 3. A broken sun (or a sun) is odd (resp. even) if $p$ is odd (resp. even).

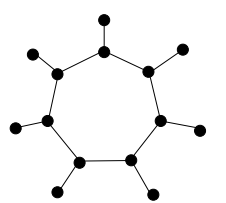

Figure 1: The sun graph $\mathrm{Sun}_{7}$.

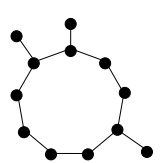

Figure 2: A broken sun belonging to $\operatorname{BSun}(9,3)$

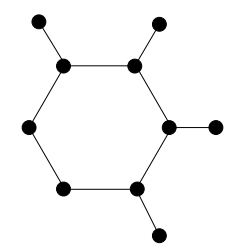

Figure 3: The consecutive broken sun CBSun $_{6,4}$

This paper is organized as follow: we first recall in Section 2 some basic and useful results on spectral graph theory. Then in Section 3 we look at the Laplacian spectrum of suns and broken suns and we show in particular that the suns and the even consecutive broken suns are determined by their Laplacian spectrum. Then in Section 4 we consider the adjacency spectrum and show that the odd suns and the odd consecutive broken suns are determined by their adjacency spectrum.

To fix notations, $Q_{G}(X)=X^{n}+a_{1} X^{n-1}+a_{2} X^{n-2}+\ldots+a_{n}$ denotes the characteristic polynomial of the adjacency matrix of a graph $G, \operatorname{Sp}(G)$ denotes the associated spectrum and $\lambda(G)$ denotes the spectral radius of the adjacency matrix of the graph $G$. A $p$-cycle is denoted by $C_{p}$ and the complete graph on two vertices is denoted by $K_{2}$.

The line graph $\mathcal{L}(G)$ of a graph $G$ has the edges of $G$ as its vertices and two vertices of $\mathcal{L}(G)$ are adjacent if and only if the corresponding edges in $G$ are incident on a common vertex.

\section{Known Results}

Here are some known results about the Laplacian spectrum of a graph.

Theorem 1 [11] Let $G=(V, E)$ be a graph where $V$ (resp. E) is the set of vertices (resp. edges). Let $\mu$ be the spectral radius of the Laplacian of $G$ and we denote by $d(v)$ the degree of a vertex $v$. We have:

$$
\max \{d(v), v \in V\}<\mu \leq \max \{d(u)+d(v), u v \in E\}
$$

Theorem 2 [6] Let $G$ be a graph, the following can be deduced from the spectrum of the Laplacian matrix of $G$ :

- The number of vertices.

- The number of edges.

- The number of connected components. 
- The number of spanning trees.

\section{Proposition 1 [2]}

i) The sum of squares of the degrees of a graph $G$ can be deduced from its Laplacian spectrum.

ii) The sum of cubes of the degrees of a graph $G$ can be deduced from its Laplacian spectrum and from the number of triangles contained in $G$.

Theorem 3 [7] Let $G$ be a bipartite graph with $n$ vertices, let $\mu_{1}(G) \geq \mu_{2}(G) \geq$ $\ldots \geq \mu_{n}(G)$ be the Laplacian eigenvalues of $G$ and $\lambda_{1}(\mathcal{L}(G)) \geq \lambda_{2}(\mathcal{L}(G)) \geq \ldots \geq$ $\lambda_{n}(\mathcal{L}(G))$ be the adjacency eigenvalues of the line graph of $G$. Then $\mu_{i}(G)=$ $\lambda_{i}(\mathcal{L}(G))+2$ for $1 \leq i \leq n-1$.

Here are some known results about the adjacency spectrum of a graph.

Theorem 4 [9] If $H$ is an induced subgraph of $G$ then $\lambda(H) \leq \lambda(G)$.

Theorem 5 [8] Let $G$ be a graph with maximum degree $d_{M}$ then $\lambda(G) \geq \sqrt{d_{M}}$.

Proposition 2 [1] $\forall p \geq 3, \lambda\left(\operatorname{Sun}_{p}\right)=1+\sqrt{2}$.

A closed walk of length $k$ is a sequence of graph vertices $v_{0}, v_{1}, v_{2}, \cdots, v_{k-1}, v_{0}$ such that $v_{i}$ and $v_{i+1}$ are adjacent and $v_{n-1}$ is adjacent to $v_{0}$. The following proposition is a classical result:

Proposition 3 Let $G$ be a graph and $\lambda_{i}, i=1, \cdots, n$ be its adjacency eigenvalues. The number of closed walks of length $k \geq 2$ in $G$ is $\sum \lambda_{i}^{k}$.

Proposition 4 [5] Let $G$ be a graph with $n$ vertices, $m$ edges and $c_{4}$ 4-cycles. Let $n_{k}$ be the number of vertices of degree $k$. Then $\sum_{\lambda_{i} \in S p(G)} \lambda_{i}^{4}=8 c_{4}+$ $\sum_{k} k n_{k}+4 \sum_{k>0} \frac{n_{k}\left(n_{k}-1\right)}{2} n_{k}$.

Theorem 6 [1] $\forall G \in B S(p, q), G \neq$ CBSun $_{p, q}, \lambda(G)<\lambda\left(C B S u n_{p, q}\right)$.

Theorem 7 [4] We call an elementary figure the complete graph on two vertices or the $q$-cycles, $q>0$. We call a basic figure $U$ every graph all of whose connected components are elementary figures. Let $p(U)$ be the number of connected components of $U$ and $c(U)$ the number of cycles in $U$. We note $\mathcal{U}_{i}$ the set of basic figures with $i$ vertices of $G$. Then the coefficient $a_{i}$ of $Q_{G}$ is:

$$
a_{i}=\sum_{U \in \mathcal{U}_{i}}(-1)^{p(U)} 2^{c(U)}, i=1,2, \ldots, n
$$

Straightforward consequences of this theorem are:

- $a_{1}=0$ since there are no basic figures with only one vertex,

- $a_{2}$ is the number of edges since a basic figure on 2 vertices is necessarily an edge (i.e. a complete graph on two vertices),

- $a_{3}$ is the number of triangles since a basic figure on 2 vertices is necessarily a triangle. 
The following theorem is also a consequence of Theorem 7 the key point of the proof is to notice that a basic figure with an odd number of vertices inevitably contains an odd cycle.

Theorem 8 [4] The length of the shortest odd cycle in $G$ and the number of such cycles are given by the smallest odd index $p$ such that $a_{p} \neq 0$.

It ensues that a graph is bipartite if and only if its adjacency spectrum is symmetric.

\section{Characterizations of suns and broken suns by means of Laplacian spectrum}

Proposition 5 A graph $L$-cospectral with $H \in B S(p, q)$ is connected, unicyclic and the size of the cycle is $p$.

Proof : We apply Theorem 2 and we note that a connected graph with as many edges as vertices is unicyclic and the number of spanning trees gives the length of the cycle.

The following proposition follows from Theorem 1]

Proposition 6 Let $G$ be a graph L-cospectral with $H \in B S(p, q)$, then the maximum degree of $G$ is lower than or equal to 5 .

Theorem 9 Let $G$ be a graph $L$-cospectral with $H \in B S(p, q)$ for $p>2$ and $1 \leq q \leq p$ then $G \in B S(p, q)$.

Proof : The first step of the proof is to determine the degree distribution of $G$. Let $n_{i}$ be the number of vertices of degree $i, i=1, \cdots, 5$ of $G$. The spectrum determines $\sum d_{i}, \sum d_{i}^{2}$ and $\sum d_{i}^{3}$ and for the graph $H$ we have $\sum d_{i}=$ $q+2(p-q)+3 q, \sum d_{i}^{2}=q+4(p-q)+9 q$ and $\sum d_{i}^{3}=q+8(p-q)+27 q$; so:

$$
\left\{\begin{aligned}
n_{1}+n_{2}+n_{3}+n_{4}+n_{5} & =p+q \\
n_{1}+2 n_{2}+3 n_{3}+4 n_{4}+5 n_{5} & =2 p+2 q \\
n_{1}+4 n_{2}+9 n_{3}+16 n_{4}+25 n_{5} & =4 p+6 q \\
n_{1}+8 n_{2}+27 n_{3}+64 n_{4}+125 n_{5} & =8 p+20 q
\end{aligned}\right.
$$

Since the Vandermonde matrix is invertible, this linear system is uniquely solvable. We have:

$$
\left\{\begin{aligned}
n_{1}+n_{2}+n_{3}+n_{4}+n_{5} & =p+q \\
n_{2}+2 n_{3}+3 n_{4}+4 n_{5} & =p+q \\
2 n_{3}+6 n_{4}+12 n_{5} & =2 q \\
6 n_{4}+24 n_{5} & =0
\end{aligned}\right.
$$

Solving this system gives $n_{4}=n_{5}=0, n_{3}=q, n_{2}=p-q$ and $n_{1}=q$. If there are $q^{\prime}<q$ vertices of degree 3 belonging to the $p$-cycle, then $n_{2} \geq p-q^{\prime}>p-q$, a contradiction. As a result there are $q$ vertices of degree 3 belonging to the $p$-cycle and $G \in B S(p, q)$. 


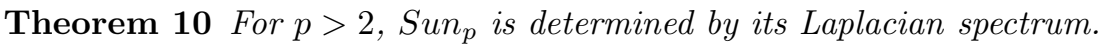

Proof : We just apply the previous Theorem and notice that $B S(p, p)=$ $\left\{\right.$ Sun $\left._{p}\right\}$.

Lemma 1 For $p \in \mathbb{N} \backslash\{0,1,2,3,4,5,7\}$ and $1<q<p-1$, let $G$ be a graph in $B S(p, q)$ and non-isomorphic to $C B S_{p, q}$, we have (we remind that $\delta_{p}^{6}$ equals 1 if $p=6$ and 0 otherwise):

$$
\sum_{\lambda_{i} \in S p(\mathcal{L}(G))} \lambda_{i}^{7}<\sum_{\lambda_{i} \in S p\left(\mathcal{L}\left(C B S_{p, q}\right)\right)} \lambda_{i}^{7}=686 q-392+14 q \delta_{p}^{6} .
$$

where $\mathcal{L}(G)$ is the line graph of $G$ and $\lambda_{i}$ denotes the adjacency eigenvalues.

Proof : The aim of the proof is to obtain a formula for $\sum_{\lambda_{i} \in \operatorname{Sp}(H)} \lambda_{i}^{k}$ where $H$ is an arbitrary graph. Once this formula established, we apply it for $k=7$ to $\mathcal{L}(G)$ and to $\mathcal{L}\left(C B S_{p, q}\right)$.

Let $M$ be a graph and let $k>1$ be an integer. A $k$-covering closed walk in $M$ is a closed walk of length $k$ in $M$ running through all the edges of $M$ at least once. Let $H$ be a graph, $M(H)$ denotes the set of all distinct subgraphs (not necessarily induced) of $G$ isomorphic to $M$ and $|M(H)|$ is the number of elements of $M(H)$. The number of $k$-covering closed walks in $M$ is denoted by $w_{k}(M)$ and we define the set $\mathcal{M}_{k}=\left\{M, w_{k}(M)>0\right\}$. As a consequence, the number of closed walks of length $k$ in $H$ is:

$$
\sum_{\lambda_{i} \in \operatorname{Sp}(H)} \lambda_{i}^{k}=\sum_{M \in \mathcal{M}_{k}} w_{k}(M)|M(H)|
$$

As an odd closed walk necessarily runs through an odd cycle, it is clear that if $M \in \mathcal{M}_{7}$ is a subgraph of $\mathcal{L}(G)$ or $\mathcal{L}\left(C B S_{p, q}\right)$ then $M$ contains one and only one triangle or $M$ contains one and only one 7-cycle. Only the graphs $T, T_{1}, T_{2}, T_{3}, T_{4} \in \mathcal{M}_{7}$ depicted in Figure 4 and the 7 -cycle $C_{7}$ can arise as subgraphs of $\mathcal{L}(G)$ and $\mathcal{L}\left(C B S_{p, q}\right)$; the 7-cycle can arise if and only if $p=6$.

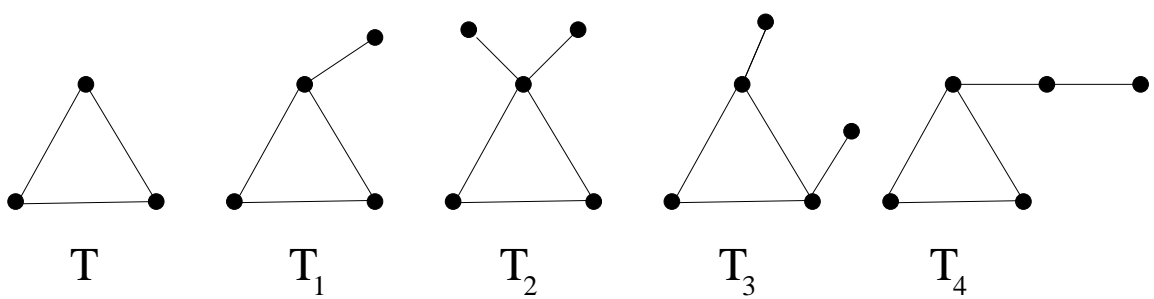

Figure 4: Subgraphs of $\mathcal{L}(G)$ and $\mathcal{L}\left(C B S_{p, q}\right)$ belonging to $\mathcal{M}_{7}$ 
Let $A, A_{i}$ denote the adjacency matrices of $T, T_{i}$. The resolution of the equations

$$
\begin{aligned}
\operatorname{tr}\left(A^{7}\right) & =w_{7}(T) \\
\operatorname{tr}\left(A_{1}^{7}\right) & =w_{7}(T)+w_{7}\left(T_{1}\right) \\
\operatorname{tr}\left(A_{2}^{7}\right) & =w_{7}(T)+2 w_{7}\left(T_{1}\right)+w_{7}\left(T_{2}\right) \\
\operatorname{tr}\left(A_{3}^{7}\right) & =w_{7}(T)+2 w_{7}\left(T_{1}\right)+w_{7}\left(T_{3}\right) \\
\operatorname{tr}\left(A_{4}^{7}\right) & =w_{7}(T)+w_{7}\left(T_{1}\right)+w_{7}\left(T_{4}\right)
\end{aligned}
$$

yields

$$
\begin{aligned}
w_{7}(T) & =126 \\
w_{7}\left(T_{1}\right) & =84 \\
w_{7}\left(T_{2}\right) & =28 \\
w_{7}\left(T_{3}\right) & =14 \\
w_{7}\left(T_{4}\right) & =14
\end{aligned}
$$

For the graph $\mathcal{L}\left(C B S_{p, q}\right), q<p-1$, we have:

$$
\begin{aligned}
\left|T\left(\mathcal{L}\left(C B S_{p, q}\right)\right)\right| & =q \\
\left|T_{1}\left(\mathcal{L}\left(C B S_{p, q}\right)\right)\right| & =4(q-2)+2 \times 3 \\
\left|T_{2}\left(\mathcal{L}\left(C B S_{p, q}\right)\right)\right| & =2(q-2)+2 \\
\left|T_{3}\left(\mathcal{L}\left(C B S_{p, q}\right)\right)\right| & =4(q-2)+2 \times 2 \\
\left|T_{4}\left(\mathcal{L}\left(C B S_{p, q}\right)\right)\right| & =8(q-4)+2 \times 7+2 \times 5
\end{aligned}
$$

Moreover if $p=6$ we have $\left|C_{7}\left(\mathcal{L}\left(C B S_{p, q}\right)\right)\right|=q$ and $w_{7}\left(C_{7}\right)=14$. So

$$
\sum_{\lambda_{i} \in \operatorname{Sp}\left(\mathcal{L}\left(C B S_{p, q}\right)\right)} \lambda_{i}^{7}=\sum_{M \in \mathcal{M}_{7}} w_{7}(M)\left|M\left(\mathcal{L}\left(C B S_{p, q}\right)\right)\right|=686 q-392+14 q \delta_{p}^{6} .
$$

We call a chain of triangles the line graph of a centipede1 (see Figure 5 for an example) and we define the length of a chain of triangles as the number of triangles.

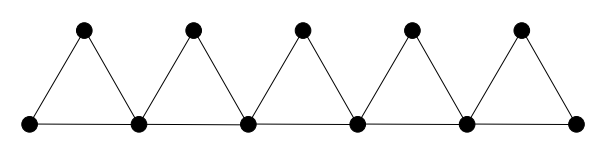

Figure 5: A chain of triangles of length 5.

Let $l_{1}, l_{2}, \cdots l_{r}$ be the maximal lengths of the chains of triangles contained in $\mathcal{L}(G)$; we have $\sum l_{i}=q$ and:

$$
\begin{aligned}
& |T(\mathcal{L}(G))|=\sum l_{i}=q \\
& \left|T_{1}(\mathcal{L}(G))\right|=\sum\left(4\left(l_{i}-2\right)+2 \times 3\right)=4 q-2 r \\
& \left|T_{2}(\mathcal{L}(G))\right|=\sum\left(2\left(l_{i}-2\right)+2\right)=2 q-2 r \\
& \left|T_{3}(\mathcal{L}(G))\right|<\sum 4 l_{i}=4 q \\
& \left|T_{4}(\mathcal{L}(G))\right|<\sum 8 l_{i}=8 q \\
& \left|C_{7}(\mathcal{L}(G))\right|=q \delta_{p}^{6}
\end{aligned}
$$

\footnotetext{
${ }^{1} \mathrm{~A}$ centipede is a tree constructed by appending a pendant vertex to each vertex of degree 2 of a path 2
} 
So

$$
\sum_{\lambda_{i} \in \operatorname{Sp}(\mathcal{L}(G))} \lambda_{i}^{7}=\sum_{M \in \mathcal{M}_{7}} w_{7}(M)|M(\mathcal{L}(G))|<686 q-224 r+14 q \delta_{p}^{6}
$$

As $r \geq 2$ we have

$$
\sum_{\lambda_{i} \in \operatorname{Sp}(\mathcal{L}(G))} \lambda_{i}^{7}<\sum_{\lambda_{i} \in \operatorname{Sp}\left(\mathcal{L}\left(C B S_{p, q}\right)\right)} \lambda_{i}^{7}
$$

Theorem 11 For $p>2$ even and $0<q<p$, the consecutive broken sun $C B S_{p, q}$ is determined by its Laplacian spectrum.

Proof : $\quad$ Let $G$ be a graph cospectral with $C B S_{p, q}, p>2$ even and $0<q<p$. According to Theorem 9, $G \in B S(p, q)$. If $q=1$ or $q=p-1$ then $|B S(p, q)|=1$ and $G$ is isomorphic to $C B S_{p, q}$. We now assume that $1<q<p-1$. If $p=4$ then we easily compute that the two graphs belonging to $B S(4,2)$ are not Laplacian-cospectral (by comparing their spectral radii for instance), thus $G$ is isomorphic to $C B S_{p, q}$. If $p \geq 6$, since $p$ is even, $G$ and $C B S(p, q)$ are bipartite, Theorem 3 implies that $\mathcal{L}(G)$ and $\mathcal{L}\left(C B S_{p, q}\right)$ have the same adjacency spectrum. If $G$ is not isomorphic to $C B S_{p, q}$ then the previous lemma gives $\sum_{\lambda_{i} \in \operatorname{Sp}(\mathcal{L}(G))} \lambda_{i}^{7}<\sum_{\lambda_{i} \in \operatorname{Sp}\left(\mathcal{L}\left(C B S_{p, q}\right)\right)} \lambda_{i}^{7}$ and $G$ cannot be cospectral with $C B S_{p, q}$, contradiction. As a result $G$ is isomorphic to $C B S_{p, q}$.

\section{Characterizations of odd suns and odd broken suns by means of adjacency spectrum}

Lemma 2 Let $G$ be a graph $A$-cospectral with $H \in B S(p, q), p$ odd and $q \leq p$. Then:

i) the coefficient $a_{p^{\prime}}, p^{\prime}$ odd, $p^{\prime} \neq p$, of $Q_{G}(X)$ is zero,

ii) the coefficient $a_{p}$ of $Q_{G}(X)$ is non-zero,

iii) the length of the shortest odd cycle of $G$ is $p$ and $G$ has one and only one p-cycle.

Proof :

i) We apply Theorem 7, As $p^{\prime}$ is odd, a basic figure with $p^{\prime} \neq p$ vertices necessarily contains an odd cycle, it becomes clear that there are no such basic figures in $H$.

ii) The length of the smallest odd cycle of $H$ is $p$ and we apply Theorem 8

iii) We apply Theorem 8 ,

Lemma 3 i) Let $G$ be a graph $A$-cospectral with $H \in B S(p, q)$, $p$ odd and $q \leq p$. $A$ connected component of $G$ different from an single vertex cannot be bipartite. ii) Let $G$ be a graph without isolated vertices and $A$-cospectral with $H \in B S(p, q)$, $p$ odd and $q \leq p$. Then $G$ is connected and unicyclic. 
Proof : i) Let $\lambda$ be a non-zero eigenvalue of the adjacency matrix of $G$. By Lemma 2 we have $Q_{G}(-\lambda)=(-1)^{n}\left(Q_{G}(\lambda)+2 a_{p} \lambda^{p}\right) \neq 0$ and so $Q_{G}(-\lambda) \neq 0$. We deduce that if $\lambda \neq 0$ is an eigenvalue of $G$ then $-\lambda$ is not an eigenvalue of $G$. A connected component of $G$ different from an single vertex cannot be bipartite because there would exist an eigenvalue $\lambda$ such that $-\lambda$ is also an eigenvalue (the spectrum of a bipartite graph is symmetric)

ii) According to i), each connected component of $G$ has an odd-cycle. But the length of the shortest odd cycle of $G$ is $p$ and $G$ has one and only one $p$-cycle (Lemma 2), moreover $|G|=p+q \leq 2 p$. It ensues that $G$ cannot have more than one connected component.

Lemma 4 If $G$ is $A$-cospectral with $H \in B S(p, q)$, then $\lambda(G) \leq 1+\sqrt{2}$ and $\Delta(G) \leq 5$. 2.

Proof : A direct consequence of Theorem 4, Theorem 5 and Proposition

Lemma 5 Let $G$ be a graph without isolated vertices and $A$-cospectral with $H \in$ $B S(p, q) p$ odd and $q \leq p$. Then there are no vertices at distance $d>1$ from the cycle and a vertex of $G$ is at distance 1 from the $p$-cycle if and only if it is a pendant vertex.

Proof : Since $C_{p} \cup K_{2}$ is not a subgraph of $G$ (Theorem 7 and Lemma 2), there are no vertices at distance $d>1$ from the cycle. It involves that a pendant vertex is at distance 1 from the cycle and that a vertex at distance 1 from the cycle is necessarily a pendant vertex (remember that $G$ is unicyclic, Lemma 31)

We can state the following theorem:

Theorem 12 Let $p$ be odd and $G$ be a graph without isolated vertices and $A$ cospectral with $H \in B S(p, q)$. Then $G \in B S(p, q)$.

Proof : The graph $G$ is connected (Lemma 3), unicyclic with a $p$-cycle (Theorem 8 and Lemma 2) and each pendant vertex of $G$ is at distance 1 from the $p$-cycle (Lemma 51).

It remains to show that the vertices of $G$ belonging to the $p$-cycle have a degree lower than or equal to 3 . The maximum degree of $G$ is lower than or equal to 5 (Lemma 4). Let $n_{i}$ be the number of vertices of $G$ of degree $i$, $i=1, \cdots, 5$ and let $n=|G|=p+q$. We have $n_{1}+n_{2}+n_{3}+n_{4}+n_{5}=n$ and $n_{1}+2 n_{2}+3 n_{3}+4 n_{4}+5 n_{5}=2 n$ ( The sum of the degrees is twice the number of edges). Since the graph $G$ is unicyclic with an odd cycle, it does not possess a 4 -cycle.

Proposition 4 gives: $\sum_{\lambda_{i} \in \mathrm{Sp} G} \lambda_{i}^{4}=2 n+4\left(n_{2}+3 n_{3}+6 n_{4}+10 n_{5}\right)$. Since $\sum_{\lambda_{i} \in \operatorname{Sp} G} \lambda_{i}^{4}=\sum_{\lambda_{i} \in \operatorname{Sp} H} \lambda_{i}^{4}=2 n+4((p-q)+3 q)$ then $n_{2}+3 n_{3}+6 n_{4}+10 n_{5}=$ $p+2 q$.

As a result we obtain: 


$$
\left\{\begin{aligned}
n_{1}+n_{2}+n_{3}+n_{4}+n_{5} & =n \\
n_{1}+2 n_{2}+3 n_{3}+4 n_{4}+5 n_{5} & =2 n \\
n_{2}+3 n_{3}+6 n_{4}+10 n_{5} & =p+2 q
\end{aligned}\right.
$$

And we deduce the following equations:

$$
\begin{gathered}
n_{2}=3 n_{4}+8 n_{5}+(p-q) \\
n_{3}+3 n_{4}+6 n_{5}=q
\end{gathered}
$$

But the vertices of $G$ of degree strictly greater than 1 are exactly the vertices belonging to the $p$-cycle (Lemma 5 ), so $n_{2}+n_{3}+n_{4}+n_{5}=p$, and using equation (3) we obtain $n_{2}=2 n_{4}+5 n_{5}+(p-q)$. This relation and equation (2) give $n_{4}=n_{5}=0$.

With this spectral characterization of the set of odd broken suns we can reveal some special odd broken suns determined by their adjacency spectrum.

Theorem 13 An odd sun is determined by its adjacency spectrum.

Proof : A direct application of Theorem 7 gives that the constant coefficient of $Q_{G}(X)$ is $(-1)^{p}$ and $G$ cannot have isolated vertices. Now, Theorem 12 gives that $G \in B S(p, p)$ and we conclude by noting that $|B S(p, p)|=1$ and $B S(p, p)=\left\{\right.$ Sun $\left._{p}\right\}$.

Remark 1 A simpler proof that does not use Theorem 12 can be done. Indeed, $G$ is connected (Lemma 3), unicyclic with a p-cycle (Theorem 8 and Lemma 2) and each pendant vertex of $G$ is at distance 1 from the p-cycle (Lemma 5). Moreover $G$ has a perfect matching 2 because if $G$ does not possess a perfect matching then the basic figures on $|G|$ vertices necessarily have a cycle and by Theorem 7 the constant coefficient of $Q_{G}$ is even which contradicts the fact that that the constant coefficient of $Q_{G}(X)$ is $(-1)^{p}$. We conclude that $G$ is a sun.

Remark 2 There exist even suns not determined by their adjacency spectrum. Two examples are depicted in Figure [6] and Figure 7 .
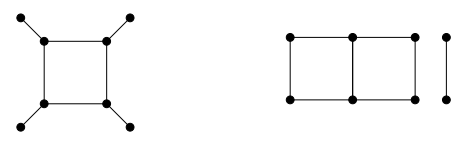

Figure 6: The graph Sun $_{4}$ and a (non-connected) graph cospectral with $\mathrm{Sun}_{4}$.
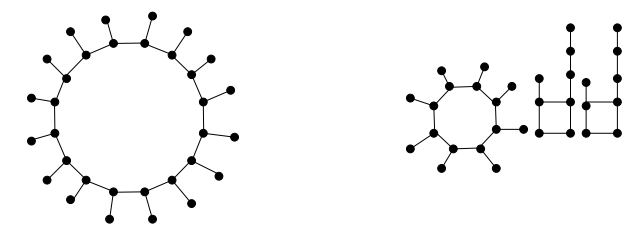

Figure 7: The graph Sun $_{16}$ and a (non-connected) graph cospectral with Sun $_{16}$.

\footnotetext{
${ }^{2} \mathrm{~A}$ perfect matching is a set of pairwise non-adjacent edges that covers all vertices of the
} graph. 
Theorem 14 The graphs $C B S u n_{p, q}, p$ odd, are determined by their adjacency spectrum.

Proof : Let $G$ be a graph $A$-cospectral with $C B S u n_{p, q}$ and let $n=p+q$ be the number of vertices of $G$.

The first step of the proof is to show that $G$ does not have isolated vertices.

If $q$ is odd then 0 is not an eigenvalue of $C B S u n_{p, q}$ because $C B S u n_{p, q}$ has one and only one basic figure with $p+q$ vertices and Theorem 7 implies that the constant coefficient of $C B S u n_{p, q}$ is non-zero. It ensues that $G$ cannot have an isolated vertex.

If $q$ is even then $C B S u n_{p, q}$ has no basic figure with $p+q$ vertices and has two basic figures with $p+q-1$ vertices and $\frac{p+q-1}{2}$ connected components, applying Theorem 7 we obtain that 0 is an eigenvalue of $C B S u n_{p, q}$ with multiplicity 1. It ensues that $G$ has at most one isolated vertex.

Let us assume that $G$ has an isolated vertex.

As a connected component of $G$ different from a single vertex cannot be bipartite (Lemma 3) it has an odd cycle. The length of the shortest odd cycle is $p$ and $|G|<2 p$ so there is one and only one connected component different from an isolated vertex. As a result $G$ is the disjoint union of an isolated vertex and a graph $G^{\prime}$ containing a $p$-cycle. Let us denote by $Q_{G}(X)=X^{n}+a_{1} X^{n-1}+$ $a_{2} X^{n-2}+\ldots+a_{n}$ the characteristic polynomial of the adjacency matrix of $G$. Since $a_{p+2}=0$ (Lemma 2), the number of $C_{p+2}$ of $G$ is equal to the number of $C_{p} \cup K_{2}$ (Theorem [7).

- If $G$ does not possess a $(p+2)$-cycle then $C_{p} \cup K_{2}$ is not a subgraph of $G^{\prime}$ which implies that each vertex not belonging to the $p$-cycle is at distance 1 of the $p$-cycle. Moreover $G$ has one and only one $p$-cycle and $G^{\prime}$ does not have $p^{\prime}$-cycles, $p^{\prime}<p, p^{\prime}$ odd, so a vertex at distance 1 from the $p$-cycle is a pendant vertex. As a result $G^{\prime}$ has one and only one cycle. Contradiction.

- Let us assume that $G$ possesses a $(p+2)$-cycle. Given that $G^{\prime}$ has $n-1$ vertices, $n$ edges and a $p$-cycle, $G^{\prime}$ has at most one $(p+2)$-cycle, so there is exactly one $C_{p} \cup K_{2}$ in $G$. With these constraints, the graph $H$ depicted in Figure 8 is an induced subgraph of $G^{\prime}$. It ensues that $G^{\prime}$ is obtained by appending pendant vertices to the vertices of $H$ belonging to the $p$ cycle. The number $q$ of vertices of $G^{\prime}$ not belonging to the $p$-cycle is $q=n_{3}+2 n_{4}+3 n_{5}$.

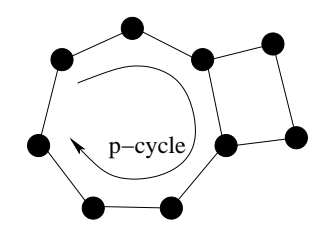

Figure 8: Graph $H$.

By Proposition 2 and Theorem 5 , the maximum degree of $G$ is lower than or equal to 5 . Let $n_{i}$ be the number of vertices of degree $i,(i=0, \cdots, 5)$, we have the following equations (the second one just says that the sum of 
the degrees is twice the number of edges):

$$
\begin{array}{r}
n=1+n_{1}+n_{2}+n_{3}+n_{4}+n_{5} \\
2 n=n_{1}+2 n_{2}+3 n_{3}+4 n_{4}+5 n_{5}
\end{array}
$$

which give

$$
n=-1+n_{2}+2 n_{3}+3 n_{4}+4 n_{5}
$$

Proposition 4 gives on one hand

$$
\sum_{\lambda_{i} \in \operatorname{Sp}(G)} \lambda_{i}^{4}=8+2 n+4\left(n_{2}+3 n_{3}+6 n_{4}+10 n_{5}\right)=6 n+4\left(3+n_{3}+3 n_{4}+6 n_{5}\right)
$$

and on the other and

$$
\sum_{\lambda_{i} \in \operatorname{Sp}\left(C B \operatorname{Sun}_{p, q}\right)} \lambda_{i}^{4}=2 n+4((p-q)+3 q)=6 n+4 q
$$

which implies $q=3+n_{3}+3 n_{4}+6 n_{5}$, a contradiction with $q=n_{3}+2 n_{4}+$ $3 n_{5}$.

As a conclusion $G$ does not have isolated vertices.

Now we can apply Theorem 12 and we have that $G \in B S(p, q)$. Since $\lambda(G)=\lambda\left(\right.$ CBSun $\left._{p, q}\right)$ we have that $G$ is isomorphic to $C B S u n_{p, q}$ (Theorem 6).

\section{References}

[1] F. Belardo, E.M Li Marzi, and S.K. Simić. Some results on the index of unicyclic graphs. Linear Algebra and its Applications, 416:1048-1059, 2006.

[2] R. Boulet. The centipede is determined by its Laplacian spectrum. C. R. Acad. Sci. Paris, Ser. I, 346, 2008.

[3] R. Boulet and B. Jouve. The lollipop graph is determined by its spectrum. Electron. J. Combin., 15(1), 2008.

[4] D. Cvetković, M. Doob, and H. Sachs. Spectra of graphs, Theory and applications. Academic press, 1978.

[5] D. Cvetković and P. Rowlinson. Spectra of unicyclic graphs. Graphs and Combinatorics, 3:7-23, 1987.

[6] Edwin R. van Dam and W.H. Haemers. Which graphs are determined by their spectrum? Linear Algebra and its Applications, 373:241-272, 2003.

[7] M. Doob. Eigenvalues of graphs. In Lowell W. Beineke and Robin J. Wilson, editors, Topics in Algebraic Graph Theory, pages 30-57. Cambrdige University Press, 2004.

[8] O. Favaron, M. Mahéo, and J.-F. Saclé. Some eigenvalues properties in graphs (conjectures of Graffiti - II). Discrete Mathematics, 111:197-200, 1993. 
[9] C Godsil and G. Royle. Algebraic Graph Theory. Springer, 2001.

[10] W.H. Haemers, X. Liu, and Y. Zhang. Spectral characterizations of lollipop graphs. Linear Algebra and its Applications, 428:2405-3034, 2008.

[11] B. Mohar. The Laplacian spectrum of graphs. Graph Theory, Combinatorics, and Applications, 2:871-898, 1991. 